\title{
Note on Translations, Transliteration, Sources, and Abbreviations
}

All translations of the poetry cited in Russian, French, and German are my own, except for one instance so noted in the text. In a few cases I have translated the poems in a less literal, looser way, in order to convey the imagery and feeling of the poems for those who do not read the original language. The translators of the prose selections cited are indicated in the bibliography; if no indication of a translator is given, the translation is my own.

I have used a dual system of transliteration from the Cyrillic, following the style of the Slavic and East European Journal and the guidelines in J. Thomas Shaw's Transliteration of Modern Russian for English-Language Publications. In the text and in all discursive parts of the endnotes, I have used Shaw's "System I," which Anglicizes Russian proper names for the generalist Western reader: soft signs are omitted, the "y"-ending is used instead of "ii," "yu" replaces "iu," etc. (e.g., Balmont, Bely, Valery, Vyacheslav, Bryusov). When a particular spelling of a Russian name is widely known, that spelling is used (e.g., Mandelstam, Gumilev). When listing Russian sources in the bibliography and endnotes, I have used a more exact scholarly system to transliterate names and titles (Shaw's "System II," i.e., the Library of Congress system with the diacritical marks omitted), so as to correspond to standard usage in library catalogues. This dual system (e.g., "Valery 
Bryusov" in the discussion, but "Valerii Briusov" in the bibliographic references) allows greater precision for the specialist and greater readability for the generalist.

The Cyrillic alphabet is used in both the text and endnotes when citing poems, concepts, and collections of poems, except in those cases when transliteration is necessary to the analysis of sounds. The Cyrillic is transliterated in the endnotes when referring to the titles of books, journals, and articles.

To save space, endnotes are given in short form with abbreviated titles. The reader is directed to the bibliography at the end of the book for complete citations of all secondary sources.

\section{SOURCES FOR POEMS CITED}

All poems cited are from the following sources unless otherwise indicated in a footnote. Poem citations in the text are followed by the date of composition (if known, otherwise date of publication), and then by the volume and page numbers in the following editions:

Akhmatova, Anna. Sobranie sochinenii v shesti tomakh. Ed. N. V. Koroleva and S. A.

Kovalenko. Moscow: Ellis Lak, 1998-2002.

Annenskii, Innokentii. Izbrannye proizvedeniia. Ed. A. V. Fedorov. Leningrad: Khudozhestvennaia literatura, 1988.

Bal'mont, Konstantin. Izbrannoe. Ed. V. Bal'mont and R. Pomirchii. Introduction by

Lev Ozerov. Moscow: Khudozhestvennaia literatura, 1980. (Note: only one poetry citation is from this edition and is labeled "Pomirchii"; the remaining citations are from the below edition.)

- Stikhotvoreniia. Ed. V. Orlov. Biblioteka poeta. Leningrad: Sovetskii pisatel', 1969. (Note: all poetry citations are from this edition except in one instance as noted above.)

Baudelaire, Charles. Oeuvres complètes. Ed. Claude Pichois. Paris: Gallimard, 1975.

Belyi, Andrei. Krylataia dusha: Stikhotvoreniia. Poema. Ed. I. A. Kuramzhina. Mir poezii. Moscow: Letopis', 1998.

Blok, Aleksandr. Polnoe sobranie sochinenii i pisem v dvadtsati tomakh. Pushkinskii

Dom/Gorky Institute. Commentary by Z. G. Mints and A. V. Lavrov. Moscow: Nauka, 1997.

Briusov, Valerii. Sobranie sochinenii v semi tomakh. Ed. P. G. Antokol'skii et al. Moscow: Khudozhestvennaia literatura, $1973^{-75}$.

Doolittle, Hilda. Collected Poems, I9I2-1944. Ed. Louis Martz. New York: New Directions, 1983 .

Eliot, T. S. Collected Poems, 1909-1962. New York: Harcourt Brace, 1963. Gautier, Théophile. Poésies complètes. Ed. René Jasinski. 3 vols. Paris: A. G. Nizet, 1970. 
Gippius, Zinaida. Stikhotvoreniia. Zhivye litsa. Ed. N. A. Bogomolov. Moscow: Khudozhestvennaia literatura, 1991.

Gumilev, Nikolai. Stikhotvoreniia i poemy. Ed. M. D. El'zon. Novaia biblioteka poeta. St. Petersburg: Akademicheskii proekt, 2000.

Hofmannsthal, Hugo von. Gesammelte Werke in zehn Einzelbänden. Ed. Bernd Schoeller and Rudolf Hirsch. Frankfurt: Fischer Taschenbuch, 1979.

Ivanov, Viacheslav. Sobranie sochinenii. Ed. O. Deschartes and D. V. Ivanov. Brussels: Foyer Oriental Chrétien, 197I.

Khlebnikov, Velimir. Sobranie Sochinenii v shesti tomakh. Ed. E. R. Arenzon and R. V. Duganov. Moscow: IMLI RAN, Nasledie, 2000-2003.

Loy, Mina. The Lost Lunar Baedeker: Poems. Ed. Roger Conover. New York: Farrar Straus \& Giroux, 1996.

Maiakovskii, Vladimir. Izbrannoe. Vsemirnaia biblioteka poezii. Rostov-on-the-Don: Feniks, 1996.

Mallarmé, Stéphane. Oeuvres. Ed. Yves-Alain Favre. Paris: Garnier, 1985.

Mandel'shtam, Osip. Sochineniia v dvukh tomakh. Ed. P. M. Nerler and A. D. Mikhailov. 2 vols. Moscow: Khudozhestvennaia literatura, 1990.

Pound, Ezra. Personae: Collected Shorter Poems. 1926. Ed. Lea Baechler and A. Walton Litz. Rev. ed. New York: New Directions, 1990.

Régnier, Henri de. La Cité des eaux. I90I. Paris: Mercure de France, I9II.

Rilke, Rainer Maria. Sämtliche Werke. 6 vols. Frankfurt: Insel, 1955-66. Reprint, Frankfurt: Insel Taschenbuch, 1987.

Samain, Albert. Oeuvres. 1924-25. Reprint, Geneva: Slatkine Reprints, 1977.

Sologub, Fiodor. Stikhotvoreniia. Ed. M. I. Dikman. Novaia biblioteka poeta. St. Petersburg: Akademicheskii proekt, 2000.

Swinburne, Algernon Charles. Poems and Ballads \& Atalanta in Calydon. Ed. Kenneth Haynes. New York: Penguin Books, 2000.

Symons, Arthur. Poetry and Prose. Selected and introduced by R. V. Holdsworth. Cheadle, England: Carcanet Press, 1974. (Note: only one poetry citation is from this edition and is labeled "Holdsworth"; the remaining citations are from the below edition.)

- Silhouettes, 1896; London Nights, 1897. Facsimile reprint, Oxford: Woodstock Books, 1993. (Note: all poetry citations are from this edition except in one instance as noted above.)

Verlaine, Paul. Oeuvres poétiques complètes. Ed. Yves-Gerard Le Dantec and Jacques Borel. Paris: Gallimard, 1962.

Whitman, Walt. Complete Poetry and Collected Prose. New York: Library of America, 1982.

Williams, William Carlos. Collected Poems. Ed. A. Walton Litz and Christopher MacGowan. New York: New Directions, 1986.

Yeats, William Butler. Collected Works. Ed. Richard J. Finneran. 2d ed. New York: Scribner, 1997. 
xiv Transliteration, Sources, Abbreviations

ABBREVIATIONS TO ANTHOLOGIES AND FREQUENTLY

\section{CITED LETTERS AND ESSAYS}

For the reader's convenience, most page references to prose excerpts by Akhmatova, Rilke, and Mandelstam refer to the widely available English translations of their prose. In some cases, particularly when translations have been altered, page references are also given to an edition of the Russian/German original. The translations of the two versions of Akhmatova's memoirs $(A A, M H)$ have occasionally been combined.

AA Polivanov, Konstantin, ed. Anna Akhmatova and Her Circle. Trans. Patricia Beriozkina. Fayetteville: University of Arkansas Press, 1994.

$A B \quad$ Pound, Ezra. $A B C$ of Reading. 1936. Reprint, New York: New Directions, 1960.

$A D$ Beckson, Karl, ed. Aesthetes and Decadents of the I8gos: An Anthology of British Poetry and Prose. Rev. ed. Chicago: Academy Chicago Publishers, I98I.

AK Bek, Tat'iana A., ed. Antologiia akmeizma. Moscow: Moskovskii rabochii, 1997.

CP Mandelstam, Osip. Complete Critical Prose. Trans. Jane Gary Harris and Constance Link. Rev. ed. Ann Arbor, Mich.: Ardis, 1997.

E Yeats, William Butler. Essays and Introductions. New York: Collier Books, 196r.

FM Apollonio, Umbro, ed. Futurist Manifestoes. Trans. R. W. Flint. New York: Viking Press, 1973.

GE Bode, Dietrich, ed. Gedichte des Expressionismus. Stuttgart: Reclam, 1966.

L Rilke, Rainer Maria. Letters, I892-rgro. Trans. Jane Bannard Greene and M. D. Herter Norton. New York: Norton, 1945.

LC Rilke, Rainer Maria. Letters on Cézanne. Ed. Clara Rilke. Trans. Joel Agee. New York: Fromm International, 1985.

LE Pound, Ezra. Literary Essays. Ed. T. S. Eliot. New York: New Directions, 1968.

LY Vietta, Silvio. Lyrik des Expressionismus. Munich: Deutscher Taschenbuch, 1976.

M Markov, Vladimir, ed. Manifesty i programmy russkikh futuristov. Slavische Propyläen, Texte in Neu- and Nachdrucken, vol. 27. Munich: Wilhelm Fink, I967.

MH Akhmatova, Anna. My Half Century: Selected Prose. Ed. Ronald Meyer. Evanston, Ill.: Northwestern University Press, 1997.

MP Aldington, Richard. "Modern Poetry and the Imagists." The Egoist $\mathrm{I}$, no. 2 (June I, I914): 201-3.

MS Miner, Earl, and Hiroko Odagiri, eds. and trans. The Monkey's Straw Raincoat and Other Poetry of the Bashō School. Princeton: Princeton University Press, 1981. 
NT Gorodetskii, Sergei. "Nekotorye techeniia v sovremennoi russkoi poezii." Apollon, January 1913, no. I: 46-50.

NTV Doolittle, Hilda. Notes on Thought and Vision \& the Wise Sappho. San Francisco: City Lights, 1982.

P Gumilev, Nikolai. Pisma o russkoi poezii. Ed. G. M. Fridlender and R. D. Timenchik. Moscow: Sovremennik, 1990.

PR Lang, Cecil Y., ed. The Pre-Raphaelites and Their Circle. Boston: Houghton Mifflin, 1968.

RA Zilboorg, Caroline, ed. Richard Aldington \& H.D.: The Early Years in Letters. Bloomington: Indiana University Press, 1992.

RF Lawton, Anna, ed. Russian Futurism Through Its Manifestoes, 19I2-I928. Ithaca, N.Y.: Cornell University Press, 1988.

RS Peterson, Ronald E., ed. The Russian Symbolists: An Anthology of Critical and Theoretical Writings. Ann Arbor, Mich.: Ardis, 1986.

$S \quad$ Mandel'shtam, Osip. Sochineniia v dvukh tomakh. Ed. P. M. Nerler and A. D. Mikhailov. Moscow: Khudozhestvennaia literatura, 1990.

SL Pound, Ezra. Selected Letters of Ezra Pound, 1907-I94I. Ed. D. D. Paige. New York: New Directions, 197I.

SP Engelberg, Edward, ed. The Symbolist Poem: The Development of the English Tradition. New York: Dutton, 1967.

SS Akhmatova, Anna. Sobranie sochinenii v shesti tomakh. Ed. N. V. Koroleva and S. A. Kovalenko. Moscow: Ellis Lak, 1998-2002.

SW Rilke, Rainer Maria. Sämtliche Werke. 6 vols. Frankfurt: Insel, 1955-66. Reprint, Frankfurt: Insel Taschenbuch, 1987.

SWP Rilke, Rainer Maria. Selected Works. Vol. I, Prose. Trans. G. Craig Houston. London: Hogarth Press, 1954.

TP Gippius, Vasilii Vasil'evich. “Tsekh poetov." In Desiatye Gody by Anna Akhmatova, ed. R. D. Timenchik and K. M. Polivanov, 82-86. Moscow: MPI, 1989.

$V \quad$ Pound, Ezra. "Vorticism." Fortnightly Review, n.s., 96 (September 1914): 461-71. 
